\title{
Workspace Analysis of 7-DOF Humanoid Robotic Arm
}

\author{
Jinliang Luo ${ }^{\mathrm{a}}$, Qun Wen ${ }^{\mathrm{b}}$, Jialai He ${ }^{\mathrm{c}}$ and Bin $\mathrm{Ye}^{\mathrm{d}}$ \\ School of Mechanical Engineering, University of South China, Hengyang 421001, China \\ aroy00123@163.com, bwen0202@126.com, chejialai@163.com, dyeb0126@163.com
}

Keywords: Monte Carlo method; 7-DOF; Workspace; Humanoid robotic arm.

\begin{abstract}
The 7-DOF humanoid robotic arm joint coordinate systems are established and link parameters are determined by D-H method, and the robotic arm kinematics model is established. The position and orientation of robotic arm end-effector is generated by homogeneous transform method. Monte Carlo method is proposed in analyzing the workspace of robotic arm and the echogram of robotic arm is calculated based on mapping relation from joint space to workspace of robotic arm. The reference basis is provided for follow-up robot trajectory planning, dynamics analysis, and motion control and parameter optimization.
\end{abstract}

\section{Introduction}

High-tech robot technology combines a variety of disciplines, such as computer, control theory, mechanisms, information and sensor technology, artificial intelligence and bionics, and it is very active in contemporary research and widely used in the fields. Compared with other robotic arms, humanoid robotic arm has a high degree of adaptability and flexibility, and it concretely performs in low requirements for environment, flexible motion and low energy consumption. Humanoid robotic arm has become one of the most active research direction in robot field and is a hot research for scholars from various countries. It will be surely the latest development trend and direction of modern manufacturing automation ${ }^{[1,2]}$.

The flexibility of robotic arm plays a vital role in its work ability. Workspace is an important kinematics index for measuring the movement flexibility degree of robotic arm, it refers to the point coordinate that robotic arm end-effector can reach in space. Currently, the solution methods about robotic arm workspace are mainly graphical method, analytical and numerical methods.

Graphic method and analytic method are restricted by the number of joints and they can't accurately describe some robotic arms. The calculated amount of numerical method is too large and the reliability can't be guaranteed for some boundary surfaces ${ }^{[3,4]}$. Monte Carlo method is used in this paper, derives from the numerical method and bases on random probability algorithm. With the rapid development of computer technology, Monte Carlo method has been more widely used ${ }^{[5]}$.

In this paper, the 7-DOF humanoid robotic arm is studied. Its kinematic model is established by $\mathrm{D}-\mathrm{H}$ method and the position and orientation of robotic arm end-effector is generated. The workspace of humanoid robotic arm is analyzed in MATLAB environment by D-H method.

\section{Structure and modeling approach}

Workspace is the robot end-effector work area that is according to robotic configuration and the size of link and wrist joint, the point set robot can reach. It is the important indicator for measuring mechanism function, and it is very significant for mechanism design, control and operate. It can be determined by mathematic equations, and these equations define the constraint conditions of robotic links and joints. Accessible workspace refers to all points set that the end-effector reference point can reach and it doesn't consider the position and orientation of end-effector.

The structure and its link parameters. The 7-DOF humanoid robotic arm consists of shoulder joint, elbow joint and wrist joint. The shoulder joint with three degrees of freedom, the elbow joint with a degree of freedom and the wrist joint with three degrees of freedom, and the structure model is 
shown in Fig.1. Shoulder joint and elbow joint are used to determine the spatial position, and wrist joint is used to determine the position and orientation of robotic arm.

D-H model describes a very simple method for establishing the model about the links and the joints of robot, and it can be used in any robotic configurations regardless of robot structure sequence and complexity. In order to describe the motion relationship of robot arm adjacent links, the kinematics model is established by the D-H method for the robotic arm shown in Fig. 1 and the robot each joint reference coordinate system is established. The coordinate system is shown in Fig. 2 and the $\mathrm{D}-\mathrm{H}$ parameters are shown in Table 1 . Where, $\theta_{i}$ is the joint variable, $\theta_{\text {imin }}$ for joint variable lower limit and $\theta_{\text {imax }}$ for joint variable ceiling, $\mathrm{d}_{i}$, and $\alpha \mathrm{i}_{-1}$ and $a_{i-1}$ are three fixed link parameters.

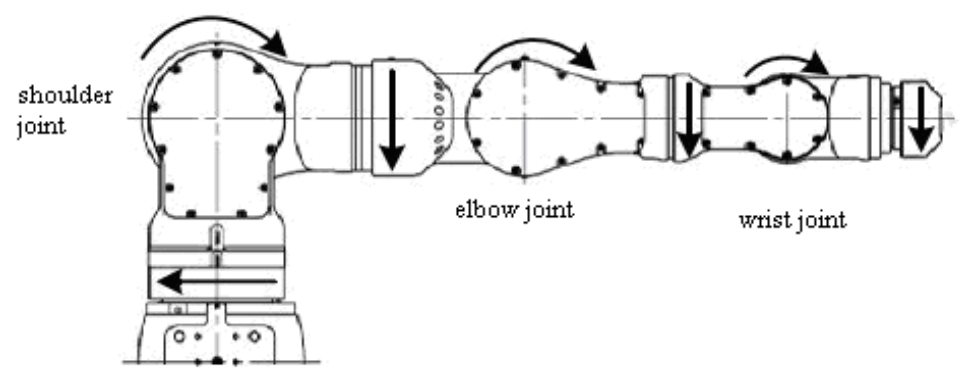

Fig. 1 The humanoid robotic arm structure model

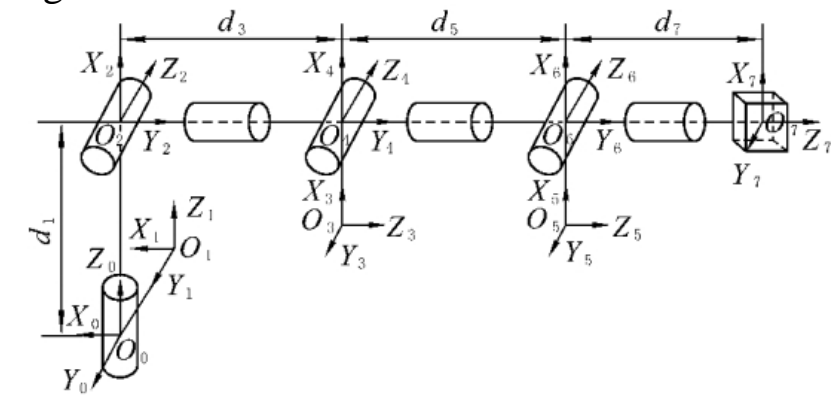

Fig. 2 The humanoid robotic arm link coordinates

Table1 The 7-DOF humanoid robotic arm D-H parameters table

\begin{tabular}{ccccccc}
\hline $\mathrm{i}$ & $\theta \mathrm{i}$ & $\mathrm{di} /(\mathrm{mm})$ & $\alpha \mathrm{i}-1 /\left(^{\circ}\right)$ & $\mathrm{ai}-1 /(\mathrm{mm})$ & $\theta \operatorname{imin} /\left(^{\circ}\right)$ & $\theta \operatorname{imax} /\left(^{\circ}\right)$ \\
\hline 1 & $\theta 1$ & $\mathrm{~d} 1=410$ & 0 & 0 & -180 & 180 \\
2 & $\theta 2$ & 0 & 90 & 0 & -110 & 110 \\
3 & $\theta 3$ & $\mathrm{~d} 3=490$ & -90 & 0 & -170 & 170 \\
4 & $\theta 4$ & 0 & 90 & 0 & -130 & 130 \\
5 & $\theta 5$ & $\mathrm{~d} 5=420$ & -90 & 0 & -180 & 180 \\
6 & $\theta 6$ & 0 & 90 & 0 & -110 & 110 \\
7 & 07 & $\mathrm{~d} 7=180$ & -90 & 0 & -180 & 180 \\
\hline
\end{tabular}

Forward kinematics analysis. Robot kinematics analysis is a prerequisite for robotics technology research and is also the foundation for robot workspace analysis and motion control. Robotic arm forward kinematics analysis is: robotic arm link geometry parameters and joint angle vectors are known, and calculating the position and orientation of robotic arm end-effectors relative to the reference coordinate system.

The position and orientation transformation matrix from robotic adjacent joint coordinate system $i-1$ to $i$ is as equation (1).

$$
{ }_{i}^{i-1} T=\left[\begin{array}{cccc}
c \theta_{i} & -s \theta_{i} & 0 & a_{i-1} \\
s \theta_{i} c \alpha_{i-1} & c \theta_{i} c \alpha_{i-1} & -s \alpha_{i-1} & -s \alpha_{i-1} d_{i} \\
s \theta_{i} s \alpha_{i-1} & c \theta_{i} s \alpha_{i-1} & c \alpha_{i-1} & c \alpha_{i-1} d_{i} \\
0 & 0 & 0 & 1
\end{array}\right]
$$


Where, $\mathrm{c} \theta_{i}=\cos \left(\theta_{i}\right), \mathrm{s} \theta_{i}=\sin \left(\theta_{i}\right), \mathrm{c} \alpha_{i-1}=\cos \left(\alpha_{i-1}\right), \mathrm{s} \alpha_{i-1}=\sin \left(\alpha_{i-1}\right)$. The position and orientation transformation matrix between humanoid robotic arms corresponding each joint coordinate system can be established by robotic arm D-H parameters in Table 1 and equation (1).

The position and orientation transformation matrix of robotic arm end coordinate system $\mathrm{O}_{7}$ relative to basis coordinate system $\mathrm{O}_{0}$ is:

$$
{ }_{7}^{0} T={ }_{1}^{0} T_{2}^{1} T_{3}^{2} T_{4}^{3} T_{5}^{4} T_{6}^{5} T_{7}^{6} T=\left[\begin{array}{cccc}
r_{11} & r_{12} & r_{13} & p_{x} \\
r_{21} & r_{22} & r_{23} & p_{y} \\
r_{31} & r_{32} & r_{33} & p_{z} \\
0 & 0 & 0 & 1
\end{array}\right]
$$

Where, the position and orientation vectors of end-effector relative to the basis coordinate system are $\left[\begin{array}{lll}r_{11} & r_{21} & r_{31}\end{array}\right]^{T},\left[\begin{array}{lll}r_{12} & r_{22} & r_{32}\end{array}\right]^{T},\left[\begin{array}{lll}r_{13} & r_{23} & r_{33}\end{array}\right]^{T}$, the position vector of end-effector relative to the basis coordinate system is $\left[\begin{array}{lll}p_{x} & p_{y} & p_{z}\end{array}\right]^{T}$.

\section{Solution and analysis}

Workspace calculation. Monte Carlo method, also called statistical simulation method, is a numerical method by means of random sampling to solve mathematical problems, it is widely used to describe certain random physical phenomena in engineering. This method is easy to implement graphical display function and calculates fast and simply, it can avoid the complicated mathematical derivation and calculation process. It is suitable for solving workspace of any joint type robotic arm without the limitation for the range of joint variables, and its error is independent of the dimension. The basic idea of the method is that robotic arm each joint works in their respective ranges and when all joint values in the corresponding range are randomly traversed, all random values set of end point constitutes workspace of robotic arm. Specific steps to solve workspace in MATLAB are as follow.

(1) According to robotic arm kinematic positive solutions, figuring out the position vector of robotic arm end-effectors relative to the basis coordinate system $\left[\begin{array}{lll}p_{x} & p_{y} & p_{z}\end{array}\right]^{T}$. Because workspace of robotic arm refers to all points set that the end-effector reference point can reach, so the position and orientation of end-effector isn't considered here.

(2) Within the range of each joint variable value, $N$ random values from 0 to 1 are generated by function rand $(\mathrm{j})$ and these random values are regarded as the random step length variables $(\mathrm{j}=1,2, \ldots$, $\mathrm{N})$, i.e. $\left(\theta_{i \max }-\theta_{i \min }\right)$ rand $(j)$, so the pseudo-random value of robotic arm joint variable is obtained, as follows.

$$
\theta_{i}=\theta_{i \min }+\left(\theta_{i \max }-\theta_{i \min }\right) \operatorname{rand}(j)
$$

Where $\theta_{\text {imin }}, \theta_{\text {imax }}$ respectively represent minimum, maximum of joint variable, and $i$ is the number of joints and selected $1 \sim 7$.

(3) Taking $\mathrm{N}$ pseudo-random values of joint variables obtained in the step (2) into the kinematic positive solution equation, and figuring out the end point corresponding position vector of robotic arm. Inorder to reflect the actual workspace of robotic arm, the coordinate point value may be more.

(4) The end point position vector value of robotic arm is proportionally displayed in the computer graphics device with plotting points, and the workspace nephogram of robotic arm is obtained, i.e. Monte Carlo workspace of robotic arm.

Workspace analysis. The workspace simulation analysis of robotic arm is done by Monte Carlo method on MATLAB.Taking the number of random coordinate points $\mathrm{N}=20000$, and workspace of the 7-DOF humanoid robotic arm is obtained, as shown in Fig.3 to Fig.6.

The workspace range of robotic arm can be obtained from Fig.4, 5 and 6. As follows, $x \in[-1100,1100] \mathrm{mm}, y \in[-1100,1100] \mathrm{mm}, z \in[-300,1500] \mathrm{mm}$. The accessible workspace of robotic arm consists of an approximate ellipsoid. The simulated workspace accords with the actual work of each joint, its size corresponds to the body design parameter of robotic arm, and its projection is 
symmetrically distributed on each plane without obvious voids. The shape of workspace is compact and the simulated results are consistent with the actual workspace.

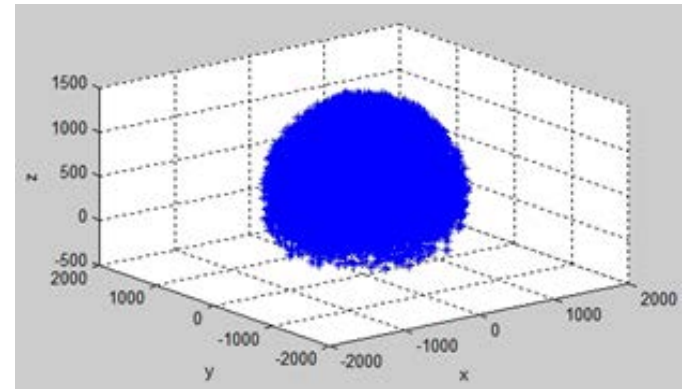

Fig.3 the accessible workspace 3-D map of robotic arm

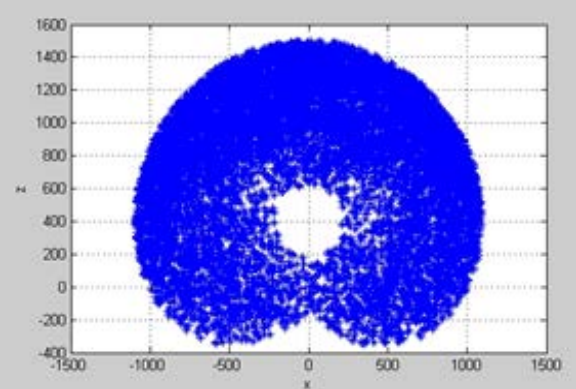

Fig.5 XOZ sectional projection

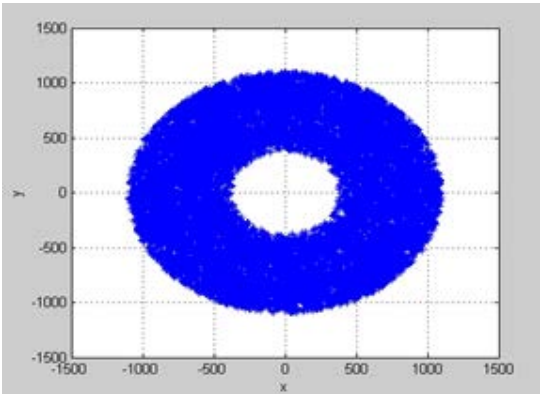

Fig.4 XOY sectional projection

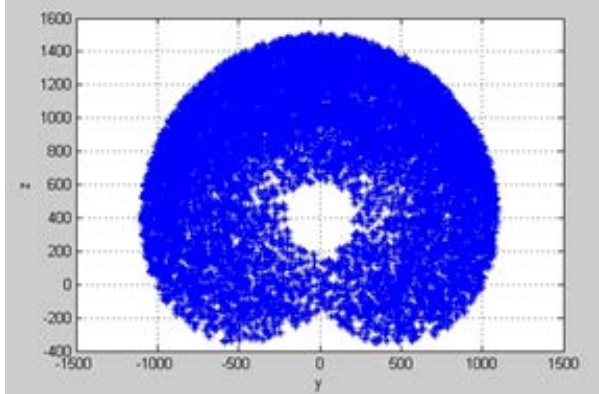

Fig.6 YOZ sectional projection

\section{Conclusions}

(1) The kinematic model of the 7-DOF humanoid robotic arm is established by D-H method. The kinematic equations of robotic arm are solved by the homogeneous transformation, and the position and orientation vectors of robotic arm end-effector are obtained.

(2) According to the mapping relation from the joint space of robotic arm to workspace, and workspace of robotic arm is figured out by Monte Carlo method in MATLAB environment. The simulated workspace is accurate and intuitive and shows preferably the actual workspace of robotic arm. So the important theoretical basis is provided for follow-up robotic arm trajectory planning, dynamics analysis, and motion control and parameter optimization.

\section{Acknowledgements}

This work was financially supported by Hunan Province S\&T plan Project (2010GK3085).

\section{References}

[1] Wang Tianmiao,Tao Yong. Research Status and Industrialization Development Strategy of Chinese Industrial Robot, J. Journal of Mechanical Engineering, 9(2014) p. 1-13.

[2] TAN Min,WANG Shuo. Research Progress on Robotics , J. ACTA Automatoca Sinica, 39(2013) p. 963-972.

[3] Tian Haibo,Ma Hongwei,Wei Juan.Workspace and Structural Parameters Analysis for Manipulator of Serial Robot Manipulator workspace analysis using the Monte Carlo method, J. Transactions of the Chinese Society for Agricultural Machinery, 44(2013) p. 196-201.

[4] Zhang Ming,He Qingzhong.The Workspace Analysis of the Articulated Palletizing Robot, J. Modular Machine Tool \& Automatic Manufacturing Technique, 7 (2013) p. 68-74.

[5] rastegar j, fardanesh b. Manipulator workspace analysis using the Monte Carlo method, J. Mechanism and Machine Theory, 25(1990) p. 233-239. 